\title{
Optimization Strategies to Reduce Alarm Fatigue in Patient Monitors
}

\author{
Mengxing Liu ${ }^{1,2}$, Zehui Sun ${ }^{1}$, Wenyu Ye ${ }^{1}$, Xianliang $\mathrm{He}^{1}$, Haoyu Jiang ${ }^{1}, \mathrm{Ye} \mathrm{Li}^{2}$, Yiyu Zhuang ${ }^{3, *}$ \\ ${ }^{1}$ Shenzhen Mindray Bio-Medical Electronics Co, Ltd, Shenzhen, China \\ ${ }^{2}$ Shenzhen Institutes of Advanced Technology, Chinese Academy of Sciences, Shenzhen, China \\ ${ }^{3}$ Sir Run Run Shaw Hospital, Zhejiang University School of Medicine, Hangzhou, China
}

\begin{abstract}
In order to ameliorate alarm fatigue, three optimization strategies are proposed to reduce false alarms and repetitive non-actionable true alarms. The four-lead arrhythmia analysis, multi-parameter fusion and intelligent threshold reminder are adopted and evaluated in multi-center clinic study. The four-lead arrhythmia analysis algorithm includes lead optimization, beat matching, detection and classification combinations. The multi-parameter fusion algorithm aggregates the information obtained from $E C G, \mathrm{SpO}_{2}$ and $\mathrm{IBP}$ wave signals. The intelligent threshold reminder can help medical staff to adjust and recover alarm limits appropriately. The results show that more than 50\% of false alarms can be reduced by the four-lead analysis and the multi-parameter fusion analysis. In some specific occasions, the intelligent threshold reminder can reduce repetitive non-actionable true alarms significantly. And no further false-negative events are generated after using the strategies in our experiments. We demonstrate that increasing the dimensionality of parametric analysis and controlling the alarm limits is beneficial for reducing alarm fatigue in intensive care units.
\end{abstract}

\section{Introduction}

Critical care clinicians rely heavily upon alarms provided by patient monitors for prompt decision-making in hospital intensive care units (ICUs). However, as a majority of current alarms are false or clinically irrelevant, alarm fatigue occurs when clinicians are insensitive to a large number of alarms. The problem has become one of ECRI's Top 10 Health Technology Hazards for the past few years [1]. Excessive insignificant alarms cause noise disturbance, quality degradation of patient care and hospital cost increase [2-3].

To address the problem, many efforts have been made to reduce false alarms. In a prior study, we identified noisy segments through signal quality assessment, and selected artifact-free channels through multi-lead analysis of electrocardiogram (ECG) signals [4-5]. Besides that, the ideas of physiological information fusion were proposed to reduce false alarms. For example, a real-time algorithm was developed to reduce falsely-calculated heart rate (HR) or pulse rate (PR) and some arrhythmia alarms using ECG and peripheral oxygen saturation $\left(\mathrm{SpO}_{2}\right)$ signals [6]. In order to apply these strategies in practice, their validity and safety performance should be proved by more clinical evidence.

Actually, repetitive threshold-crossing events account for most of the overall alarm burden. To reduce this, clinicians can adjust the alarm thresholds for individual patients. At present, there is no standard for setting the parameter threshold value of default alarm related to degree of urgency [7]. As workload and task complexity increase, nurses in ICU have less time to evaluate a growing number of parameter thresholds one by one. Even worse, the former configuration can be continued when the latter patients are monitored. Therefore, the monitoring device should remind users to change or reinstate the thresholds timely and reasonably.

In this study, we aim to achieve a high false alarm suppression rate in practice by the four-lead ECG analysis and multi-parameter fusion techniques. Based on our prior work, invasive arterial blood pressure (IBP) signals are added to correct false arrhythmia alarms and improve the accuracy of HR/PR calculation. Moreover, a new strategy of intelligent threshold reminding is proposed to reduce repetitive non-actionable alarms.

\section{Methods}

\subsection{Four-lead ECG Analysis}

As shown in Figure 1, the four-lead ECG analysis module can detect QRS complexes, calculate heart rates and identify arrhythmias. Firstly, signal resampling, bandpass filtering and quality analysis are carried out for each channel. The leads with severe noise are eliminated based on the ranking list of signal quality index. Then, the beat detection and classification of a group of matched QRS 
complexes are synthesized using each remaining lead's information. HR values and arrhythmia alarms are generated based on the synthesis QRS results. We refer interested readers to our previous work on real time arrhythmia analysis [4] for more details.

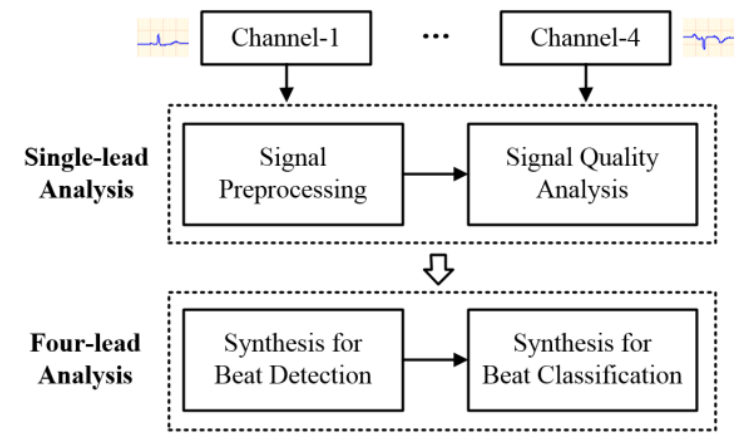

Figure 1. Schematic diagram of the four-lead ECG analysis.

Poor quality of ECG signals is strongly related to false positive alarms. Although four-lead ECG analysis can detect signal quality for each channel, some unpredictable conditions (such as temporary motion artifacts, lowamplitude signals) can also interfere beat detection and classification, resulting in false HR values and even lethal arrhythmia alarms.

\subsection{Multi-parameter Fusion}

As shown in Figure 2, we present a method to fuse $\mathrm{ECG}, \mathrm{SpO}_{2}$ and IBP signals in real time for reducing false alarms. There are four steps in this process:

(1) Pulse analysis and selection

Pulse interval, amplitude, onset and offset are acquired in real-time for each pulse both from $\mathrm{SpO}_{2}$ and IBP signals. The signal quality level associated with each pulse is generated based on the similarity between the current pulse and the recent ones. The interval of the current pulse is compared to those of the recent pulses with good quality, so as to determine whether its rhythm is regular. Low perfusion or hypotensive signals are not suitable for alarm validation.

(2) Fusion viability evaluation

It is known that, electrical activity (ECG) in heart causes changes in cardiovascular circulation $\left(\mathrm{SpO}_{2}\right.$ and IBP). When blockage or disease of peripheral vessels exists, ECG and $\mathrm{SpO}_{2}$ /IBP may lack of synchronization and thus are not suitable for fusion. Following fusion analysis will be carried out only when both signals show the same rhythm.

(3) HR and PR confirmation

This step is activated when the difference of HR and PR exceeds a predefined threshold. If half of QRS beats or pulses indicate poor signal quality, HR or PR will be considered unreliable. A heart rate value calculated by matched beats from reliable side is compared with historical trend to prove its reliability. If so, the reliable HR/PR will take place of the false PR/HR.

\section{(4) Arrhythmia validation}

When an arrhythmia alarm is triggered by the four-lead analysis module, synchronous $\mathrm{SpO}_{2} / \mathrm{IBP}$ features within a validation window are checked to determine whether the alarm should be released or rejected. For the critical alarms, the validation window is mainly determined by the starting point of the event and the time at which the alarm is generated. For others, the window is determined by the definition used for the alarm condition.

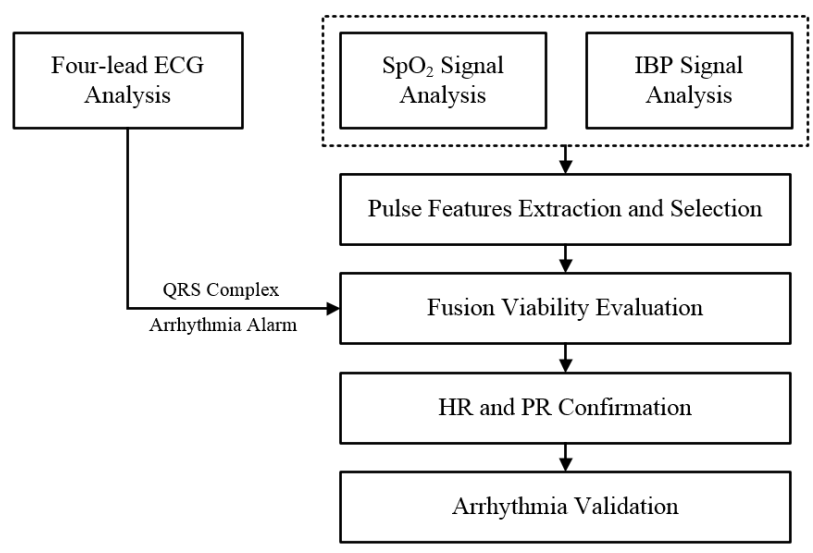

Figure 2. Schematic diagram of the multi-parameter fusion.

\subsection{Intelligent Threshold Reminder}

All the parameters of monitoring system have default threshold limits. A large number of clinically irrelevant alarms appear due to lack of personalized settings. It is found that alarm loads could be controlled by setting appropriate threshold limits for the population. However, as clinical workload increases, medical staff have less time to operate monitor system. Therefore, based on the analysis of the clinical workflow in intensive care units, the interactive function of intelligent threshold reminding is proposed to help doctors and nurses to adjust alarm limits in time.

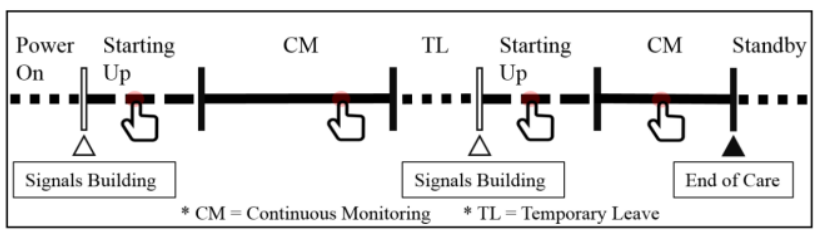

Figure 3. The monitoring workflow in ICUs.

As shown in Figure 3, from the beginning to the end of care, there are several time nodes where nurses usually observe or click on the screen of monitor system: 10 to 60 minutes after the patient is carried into the ward, and the period of nursing shift or ward round. When the alarm limit is considered unreasonable, if nurses respond to the 
reminder, an eye-catching window which contains parametric trend curve and corresponding suggestive value will pop up in front of them. The new threshold will be compared to the current alarm limits and will have an opportunity to be adjusted slightly. At the stage of starting up, the strategy trends to be conservative due to insufficient data and instable patient status. During the continuous monitoring (CM), the algorithm will check the stability of piecewise data and decide whether to make a recommendation.

\section{Results}

\subsection{Database Description}

Between October 2019 and June 2020, the ECG, $\mathrm{SpO}_{2}$, IBP and other physiological signals were collected by the Mindray $\mathrm{N}$-series patient monitors. Table 1 summarizes the clinical data of our study population from general ICUs, cardiac care units (CCUs) and neonatal intensive care units (NICUs) of 7 hospitals in China. A total of 330,294 physiological alarms were recorded from 50,304 patient-hours data. All the records were anonymised during the whole course of data collection and management. Clinical experts conducted annotations on samples of the alarm datasets for evaluation of the strategies. It should be noted that technical alarms are not discussed in this article.

Table 1. Summary of clinical database.

\begin{tabular}{cccc}
\hline Ward & Amount & Hours & Alarms \\
\hline ICU & 3 & 27,648 & 177,798 \\
CCU & 2 & 19,816 & 125,654 \\
NICU & 2 & 2,840 & 26,842 \\
Total & 7 & 50,304 & 330,294 \\
\hline
\end{tabular}

\subsection{Performance Evaluation}

In order to evaluate the performance of the three strategies separately, the collected database was sampled randomly, and then each alarm of them was confirmed by at least two clinicians.

Table 2. Sample performance of separate strategy.

\begin{tabular}{cccccc}
\hline Strategy & Sample & Types & Previous & Current & Reduction \\
\hline $\mathrm{CH}-2.1$ & $1,440 \mathrm{~h}$ & $\mathrm{~F}$ & 824 & 196 & $76.2 \%$ \\
$\mathrm{CH}-2.2$ & $1,440 \mathrm{~h}$ & $\mathrm{~F}$ & 4,256 & 1,829 & $57.0 \%$ \\
$\mathrm{CH}-2.3$ & $720 \mathrm{~h}$ & $\mathrm{NA}$ & 6,582 & 4,052 & $38.4 \%$ \\
\hline
\end{tabular}

Note: F: False alarms, NA: True but non-actionable alarms.

As shown in Table 2, the strategies proposed in chapter 2.1 2.3 were evaluated by comparing the previous results and the current results. After applying the four-lead analysis algorithm, the number of false arrhythmia and
HR alarms decreased from 824 to 196 in 1440 patienthours. About $57.0 \%$ of false alarms were reduced by using the multi-parameter fusion algorithm, wherein most of the rejected alarms are the asystole (ASY), ventricular fibrillation (VF), ventricular tachycardia (VT), abnormal IBP values caused by the motion artifacts and PR related events. If clinicians had responded to the reminder and click "OK" to accept the first recommended thresholds, the true but non-actionable alarms from 720 patient-hours multi-parameter signals might have been reduced by about $38.4 \%$. At the same time, all the methods achieved a good balance against the potential for missing clinically relevant events.

A comprehensive test was taken to investigate the performance in different parameters when the strategies were used to the clinical database. As shown in Table 3, about half of the false and non-actionable alarms can be suppressed in total.

Table 3. Performance after applying all strategies.

\begin{tabular}{ccccccc}
\hline Alarms & ECG & SpO $_{2}$ & IBP & Others & Total \\
\hline Previous & 95,668 & 58,420 & 126,842 & 49,364 & 330,294 \\
Current & 26,828 & 32,842 & 70,546 & 12,650 & 142,866 \\
Reduction & $72.0 \%$ & $43.8 \%$ & $44.4 \%$ & $74.4 \%$ & $56.7 \%$ \\
\hline
\end{tabular}

\section{Discussion}

\subsection{False Alarms}

Many ECG false alarms are usually caused by poor electrode contact, improper position of electrodes on muscles and motion artifacts. There is a strong correlation between poor quality and false alarms. Four-lead ECG analysis can achieve a good balance between interference immunity and operational quantity of algorithm. Besides that, different perspectives can be presented by adding more analysis lead, so as to improve the accuracy of wave recognition. As shown in Figure 4, the amplitudes of $\mathrm{T}$ waves are different in four leads. When we synthesize the I-lead and V-lead ECG signals, false alarms caused by towering $\mathrm{T}$-waves can be eliminated.

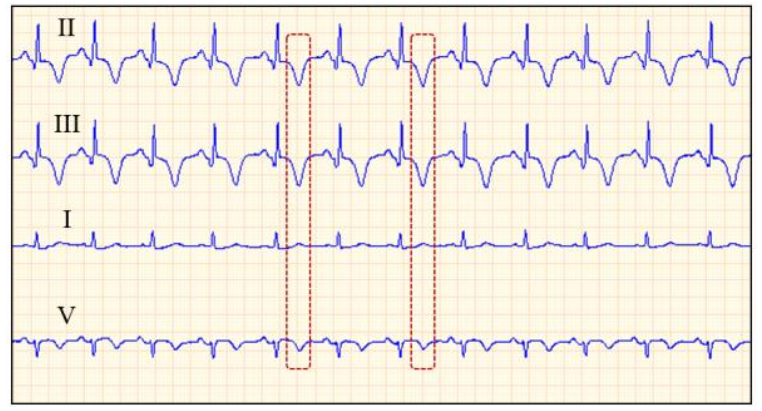

Figure 4. A segment of ECG signals with towering T-waves.

Sometimes, a large number of false ASY or VF/VT 
alarms can occur when a patient has low-amplitude ECG signals or when all leads are interfered by complex noises. As shown in Figure 5, real ASY or VF/VT events will cause inactive, feeble and disordered pulses. Since our proposed fusion algorithm can detect the pulse rhythm of $\mathrm{SpO}_{2}$ and IBP signals to validate the arrhythmias, it can effectively reduce false positive alarms. In addition, in the case of atrial fibrillation, irregular rhythm and sensor failures, false PR alarms can be corrected by ECG.

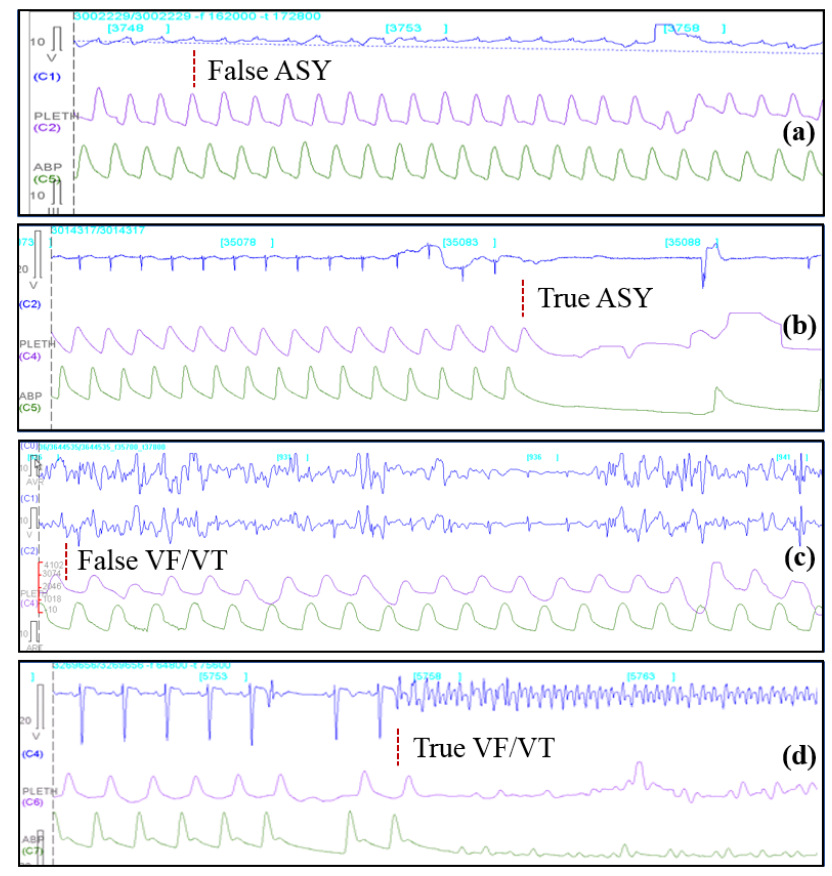

Figure 5. Four segments of multi-parameter signals: (a) False ASY; (b) True ASY; (c) False VF/VT; (d) True VF/VT.

\subsection{Clinically Irrelevant Alarms}

With the development of bio-medical technology, monitoring parameters are increasing. It is challenging to require nurses with different clinical experience to adjust the alarm limits actively according to patient status. Therefore, only by simplifying the alarm limit settings and improving the value rationality, the clinically irrelevant alarms can be reduced to the greatest extent. As shown in Figure 6, a line chart which contains 7 hours HR data is presented for a patient with coronary heart disease. Point $\mathrm{F}$ is defined as a fatigue moment when the primary nurse adjusts the low limit of HR from 40 to 35 bpm manually. Before that, 291 true but non-actionable alarms were generated in two hours. When the proposed threshold reminder was applied to the patient monitor, an automatic prompt was released at the time of 02:57:04 (marked as point A). Then, 221 alarms could be reduced without clinical risk. Therefore, the strategy is useful to reduce alarm fatigue in intensive care units.

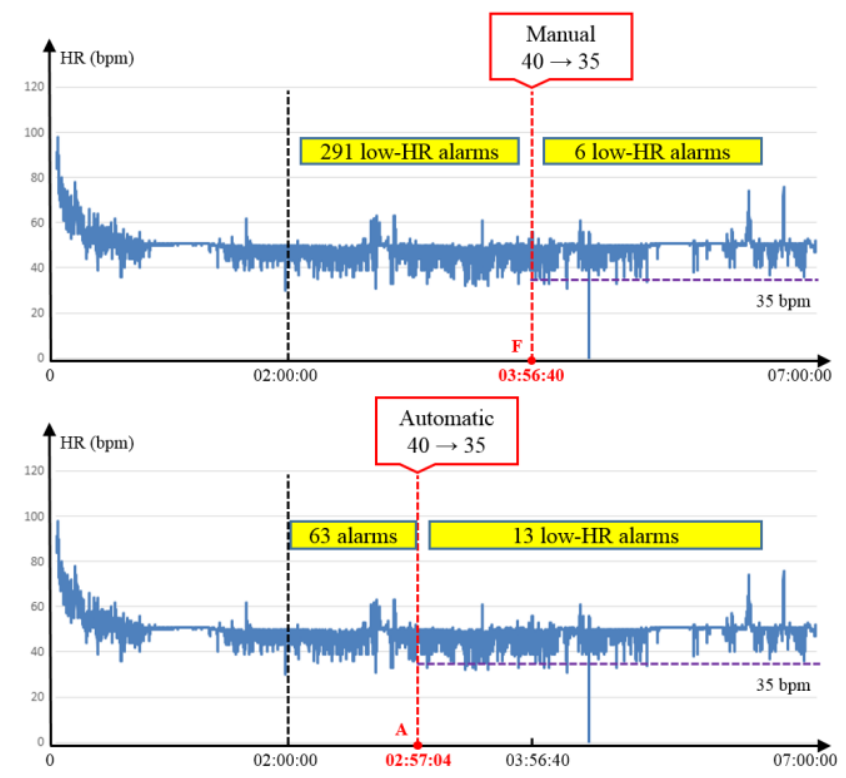

Figure 6. An example of low HR alarm limit reminder.

\section{Conclusions}

The alarm fatigue is a complex interplay of patient conditions, inappropriate user settings and algorithm deficiencies. The work presented here demonstrates that the three strategies can effectively reduce false alarms and true but non-actionable alarms without missing clinically relevant events on patient monitors.

\section{References}

[1] ECRI Institute. Top 10 health technology hazards for 2020[EB/OL]. (2019-12-20) www.ecri.org.

[2] AACN. Practice alert: alarm management [EB/OL]. (201711-22). www.ccn.aacnjournals.org.

[3] Landrigan CP. Crying wolf: false alarms and patient safety [J]. Journal of Hospital Medicine, 2015, 10(1):409-410.

[4] Zehui $\mathrm{S}$ et al. Reducing ECG alarm fatigue based on SQI analysis[C]. CinC 2014, 345-348.

[5] Jianwei $\mathrm{S}$ et al. A four-lead real time arrhythmia analysis algorithm[C]. CinC 2017, 1-4.

[6] Jianwei $\mathrm{S}$ et al. Real-time fusion of ECG and $\mathrm{SpO} 2$ signals to reduce false alarms[C]. CinC 2018, 1-4.

[7] Tabibo L et al. Epidemiology of patient monitoring alarms in the neonatal intensive care unit [J]. Journal of Perinatology, 2018, 38:1030-1038.

Address for correspondence:

Mengxing Liu

Mindray Building, Keji 12th Road South, High-Tech Park

Nanshan, Shenzhen, China

liumengxing@mindray.com 